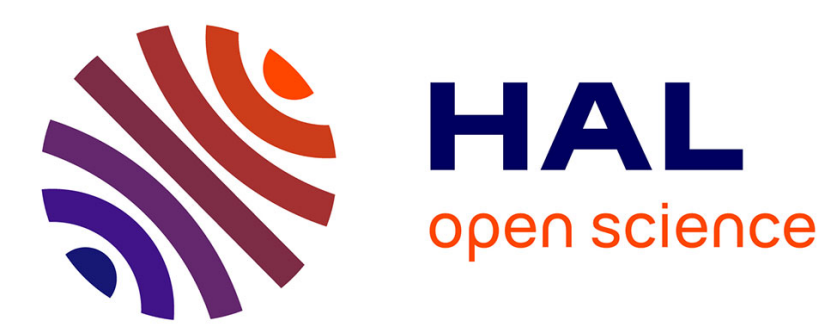

\title{
Theoretical and Numerical Analysis of a Class of Nonlinear Elliptic Equations
}

\author{
Nour Eddine Alaa, Jean Rodolphe Roche
}

\section{To cite this version:}

Nour Eddine Alaa, Jean Rodolphe Roche. Theoretical and Numerical Analysis of a Class of Nonlinear Elliptic Equations. Mediterranean Journal of Mathematics, 2005, 2, pp.327-344. 10.1007/s00009-0050048-4 . hal-00095236

\section{HAL Id: hal-00095236 https://hal.science/hal-00095236}

Submitted on 15 Sep 2006

HAL is a multi-disciplinary open access archive for the deposit and dissemination of scientific research documents, whether they are published or not. The documents may come from teaching and research institutions in France or abroad, or from public or private research centers.
L'archive ouverte pluridisciplinaire HAL, est destinée au dépôt et à la diffusion de documents scientifiques de niveau recherche, publiés ou non, émanant des établissements d'enseignement et de recherche français ou étrangers, des laboratoires publics ou privés. 


\title{
Theoretical and Numerical Analysis of a Class of Nonlinear Elliptic Equations
}

\author{
Nour Eddine Alaa and Jean Rodolphe Roche
}

\begin{abstract}
In this paper we show the existence of weak solutions for a nonlinear elliptic equations with arbitrary growth of the non linearity and data measure. A numerical algorithm to compute a numerical approximation of the weak solution is described and analyzed. In a first step a super-solution is computed using a domain decomposition method. Numerical examples are presented and commented.
\end{abstract}

\section{Introduction}

The principal objective of this work is to study existence, uniqueness and present a numerical analysis of weak solutions for the following quasi-linear elliptic problem:

$$
\left\{\begin{array}{l}
-u^{\prime \prime}(t)+G\left(t, u^{\prime}(t)\right)=F(t, u(t))+f \text { in }(0,1) \\
u(0)=u(1)=0
\end{array}\right.
$$

where $G, F:[0,1] \times \mathbb{R} \rightarrow[0,+\infty[$ are measurable and continuous with respect to $u^{\prime}$ and $u, f$ is a given finite non negative measure on $(0,1)$. Such problems arises from biological, chemical and physical systems and various methods have been proposed for study the existence, uniqueness, qualitative properties and numerical simulation of solutions(see [11], [14]). When $f$ is regular, it is proved in [12] that if (1.1) has a nonnegative super-solution in $W_{0}^{1, \infty}$ then (1.1) has a solution in $W_{0}^{1, \infty} \cap W^{2, p}$. Note that here the super-solution is required to vanish at the boundary. This provides an a priori point-wise estimate for $u^{\prime}(0)$ and $u^{\prime}(1)$. The boundedness on $u^{\prime}$ on the whole set $(0,1)$ is then obtained by a maximum principle applied to the equation satisfied by $\left|u^{\prime}\right|^{2}$. The convexity of $s \rightarrow G(t, s)$ is the essential ingredient. Many authors dealt with this problem when $f$ is irregular and $G$ is sub-quadratic with respect to $u^{\prime}$ namely:

$$
|G(t, r)| \leq c\left(g(t)+|r|^{2}\right), \quad g(t) \in L^{1}(0,1), c>0
$$

They showed that, if $G$ satisfy(1.2), (1.1) has a solution $u \in H_{0}^{1}(0,1)$ provided that (1.1) has a super-solution in $W^{1, \infty}(0,1)$ see [5], [4] and the references there in.

1991 Mathematics Subject Classification. 35J65,65N55.

Key words and phrases. Domain decomposition, Nonlinear elliptic PDE. 
The case where the super-solution itself is irregular have been treated in [2], it is a solution in $H_{0}^{1}(0,1)$ then $(1.1)$ has a solution in $H_{0}^{1}(0,1)$ provided that $G$ satisfy (1.2).

In this work we are particularly interested in situations where $f$ is irregular and where the growth of $G$ with respect to $u^{\prime}$ and $F$ with respect to $u$ are arbitrary. Let us make some precisions on model problem like:

$$
\left\{\begin{array}{l}
-u^{\prime \prime}(t)+\left|u^{\prime}(t)\right|^{q}=|u(t)|^{p}+f \text { in }(0,1) \\
u(0)=u(1)=0
\end{array}\right.
$$

where $p, q \geq 1$ and $f \in M_{B}^{+}(0,1)$, the set of nonnegative finite measure on $(0,1)$. We show here that if the semi-linear problem:

$$
\left\{\begin{array}{l}
-w^{\prime \prime}(t)=|w(t)|^{p}+f \text { in }(0,1) \\
w(0)=w(1)=0
\end{array}\right.
$$

has a solution then (1.3) has a solution. Remark here any restriction for $p$ and $q$ is imposed. For an elegant study of (1.4) one can see the work of Pierre and Baras [7]. If $w^{\prime}(0)=+\infty$ or $w^{\prime}(1)=-\infty$ then $w \notin W_{0}^{1, \infty}$ and obviously the classical approach fails to provide existence in (1.3) and new techniques have to be used. We describe some of them here.

Another approach studied here is the numerical approximation of the solution to the problem (1.1). The most important difficulties are in this approach the uniqueness and the blowup of the solution.

The general algorithm for numerical solution of these equations is one application of the Newton method to the discretized version of problem (1.1):

$$
\text { Find } U \in \mathbb{R}^{m} \text { such that } A U=H(U)
$$

where $A$ is a sparse matrix and $H: \mathbb{R}^{m} \rightarrow \mathbb{R}^{m}$ is a nonlinear operator.

The Newton algorithm is given by:

$$
\left\{\begin{array}{l}
\text { choose } U^{0} \text { in a neighborhood of the solution } \\
\text { and solve until convergence } \\
\left(A-H^{\prime}\left(U^{k}\right) I d\right)\left(U^{k+1}-U^{k}\right)=-A U^{k}+H\left(U^{k}\right)
\end{array}\right.
$$

where $H^{\prime}\left(U^{k}\right)$ is the Jacobian matrix of the operator $H$ computed in $U^{k}$ and $I d$ is a matrix of identity in $\mathbb{R}^{m}$. This method converges quadratically when it converges. Convergence depend in particular in the choice of $U^{0}$ and the existence and uniqueness of solutions of the linear system (1.6). In the case of problem (1.1) the matrix $A-H^{\prime}\left(U^{k}\right) I d$ is often singular. Consider the following example:

$$
\left\{\begin{array}{l}
-u^{\prime \prime}(t)=\alpha u(t)+\beta \text { in }(0,1) \\
u(0)=u(1)=0
\end{array}\right.
$$

where $\alpha$ and $\beta$ belongs to $\mathbb{R}$. It is easy to verify that (1.7) have an infinity of solutions when $\alpha=(2 \pi p)^{2}$. For all $B \in \mathbb{R}, \exists u_{B}$ solution of (1.7) where:

$$
u_{B}(t)=\frac{\beta}{\alpha}(1-\cos (p \pi t))+B \sin (p \pi t)
$$


If we consider a classical discretization of $u^{\prime \prime}$ by a finite differences schema and choose $\alpha$ an eigenvalues of the matrix $A$. The Newton schema is written as follows:

$$
(A-\alpha I d)\left(U^{k+1}-U^{k}\right)=-A U^{k}+H\left(U^{k}\right)
$$

Clearly the matrix $A-\alpha I d$ is singular and the system (1.9) have not necessary a solution or a infinite number of solutions if $-A U^{k}+H\left(U^{k}\right) \in \operatorname{Im}(A-\alpha \operatorname{Id})$.

To overcame this difficulty we introduce a domain decomposition to compute an approximation of $\delta u^{k}=u^{k+1}-u^{k}$ by the resolution of a sequence of problems of type (1.1) in subset $\Omega_{i}$ of $(0,1)$, such that $\Omega=\bigcup_{i=1, K} \Omega_{i}$. The idea of the method came from the following remark [17]:

Lemma 1.1. Let $0 \leq a<b \leq 1, a_{i} \in L^{\infty}(0,1)$, for $i=1,2$. If $|b-a|$ is small enough then the operator $-\frac{d^{2}}{d t^{2}}-a_{1}(t) \frac{d}{d t}-a_{2}(t) I d$ have an inverse in $(a, b)$.

We have organized this paper in the following manner. In section 2 we give the precise setting of the problem, we present an approximate equation for (1.1) and we prove that the existence of weak super-solutions implies the existence of weak solutions, without any restriction of the growth of $G$ with respect to $u^{\prime}$, this result generalize the classical result of [12], [5] and [2].

In section 3 we present an approximation scheme for problem (1.1) based on the Schwarz overlapping domain decomposition method, combined with finite element method.

\section{Mathematical analysis of the problem}

Throughout this paper we suppose $f$ is a nonnegative finite measure on $(0,1)$

and $G, F:[0,1] \times \mathbb{R} \rightarrow[0,+\infty)$ are such that $G, F$ are measurable

The functions $r \rightarrow G(t, r), F(t, r)$ are continuous a.e. $t$

$F(t,$.$) is no decreasing and G(t,$.$) is convex,$

$\forall r \in \mathbb{R}, G(., r), F(., r)$ are summable functions on $(0,1)$

$$
G(t, 0)=\min \{G(t, r), r \in \mathbb{R}\}=0 \text { and } F(t, 0)=0 .
$$

Fix $1 \leq p \leq \infty$ end let $\mathrm{k}$ be a nonnegative integer. The Sobolev spaces $W^{k, p}(0,1)$ consist of all the function $f \in L^{p}(0,1)$ such that for each index $\alpha$ with $|\alpha| \leq k, \partial^{\alpha} f$ exist in the weak sense and belongs to $L^{p}(0,1)$. If $p=2$ we usually write $W^{k, 2}(0,1)=H^{1}(0,1)$.

We denote $W_{0}^{k, p}(0,1)$ the closure of $C_{c}^{\infty}(0,1)$ in $W^{k, p}(0,1)$. The Sobolev space $W_{l o c}^{k, p}(0,1)$ is the set of all the function $f \in L_{l o c}^{p}(0,1)$ such that for all $\Omega \subset \subset(0,1)$, $\left.f\right|_{\Omega} \in W^{k, p}(0,1)$. 
Now we introduce the notion of weak solution, super-solution and sub-solution used here.

Definition 2.1. A function $u$ is said to be a weak solution of (1.1) if

$$
\left\{\begin{array}{l}
u \in W_{l o c}^{1, \infty}(0,1) \bigcap C_{0}[0,1] \\
-u "(t)+G\left(t, u^{\prime}(t)\right)=F(t, u(t))+f \text { in } \mathcal{D}^{\prime}(0,1)
\end{array}\right.
$$

(replace in $(2.6)=$ by $\geq$ for a weak super-solution and by $\leq$ for a weak sub-solution)

Remark 2.2. In (2.6) $u \in W_{l o c}^{1, \infty}(0,1)$, using $(2.4)$ we have $G\left(t, u^{\prime}(t)\right)$ and $F(t, u(t)) \in L_{l o c}^{1}(0,1)$. Hence every term in (2.6) makes sense.

This enables us to state the main result of this paper.

Theorem 2.3. Assume that (2.1)-(2.5) and $f \in M_{B}^{+}(0,1)$ hold. Assume that there exists a weak solution $\bar{w}$ for the problem,

$$
\left\{\begin{array}{c}
\bar{w} \in W_{l o c}^{1, \infty}(0,1) \cap C_{0}[0,1] \\
-\bar{w}^{\prime \prime}=F(., \bar{w})+f \quad \text { in } \mathcal{D}^{\prime}(0,1)
\end{array}\right.
$$

Then $\bar{w}$ is a super-solution of (1.1) and there exist a weak solution $u$ of (1.1) such that $u \leq \bar{w}$.

Remark 2.4. 1) It should be noted that there is not growth restriction on the lower order nonlinearity of $F$ and $G$ w.r.t. $u$ and $u^{\prime}$ respectively. Hence the present theorem extends some results in [2], [5].

2)For any finite nonnegative measure $f$, the problem:

$$
\left\{\begin{array}{l}
\underline{w} \in W_{0}^{1, \infty}(0,1), \quad \underline{w} \geq 0 \text { in }(0,1) \\
-\underline{w}^{\prime \prime}+G\left(t, \underline{w}^{\prime}\right)=f \text { in } \mathcal{D}^{\prime}(0,1)
\end{array}\right.
$$

has a unique solution $\underline{w}$, see [1], and remark here that $\underline{w}$ is a sub-solution of the problem (2.6).

\subsection{An approximate equation}

For $n \geq 0$, we consider the Yosida approximation $G_{n}(t,$.$) of G(t,$.$) defined by:$

$$
G_{n}(t, r)=\left\{\begin{array}{l}
G(t,-n)+G_{r}^{\prime}(t,-n)(r+n) \text { if } r \leq-n \\
G(t, r) \quad \text { if }|r|<n \\
G(t, n)+G_{r}^{\prime}(t, n)(r-n) \quad \text { if } r \geq n
\end{array}\right.
$$

where $G_{r}{ }^{\prime}$ denotes a section of the sub-differential of $G$ with respect to $r$.

Then $G_{n}$ satisfies (2.2) -(2.5) and

$$
G_{n} \leq G, G_{n} \leq G_{n+1}
$$

then $G_{n}(t,$.$) increases a.e. to G(t,$.$) as n$ tends to infinity.

According to the result in [1], [5] there exists a sequence $\left(u_{n}\right)$ of solution of the problem:

$$
\left\{\begin{array}{l}
u_{n+1} \in W_{0}^{1, \infty}(0,1) \\
-u_{n+1}^{\prime \prime}+G_{n+1}\left(t, u_{n+1}^{\prime}\right)=F\left(u_{n}\right)+f \text { in } \mathcal{D}^{\prime}(0,1)
\end{array}\right.
$$

where $u_{0}=\bar{w}$. 


\subsection{Estimates-Passing to the limit}

In order to proof the theorem 2.3 we propose to send $n$ to infinity in (2.11). For this we will need some estimates passing to the limit.

Lemma 2.5. Let $a(t) \in L_{l o c}^{1}(0,1), v \in W_{l o c}^{1,1}(0,1) \cap C_{0}[0,1]$ such that

$$
\left\{\begin{array}{c}
a(t) v^{\prime}(t) \in L_{l o c}^{1}(0,1) \\
-v^{\prime \prime}-a v^{\prime} \geq 0 \quad \text { in } \quad \mathcal{D}^{\prime}(0,1)
\end{array}\right.
$$

Then $v \geq 0$ in $[0,1]$.

See a proof in $[1]$

Lemma 2.6. Let $u \in W_{l o c}^{1,1}(0,1), \underline{v}, \bar{v} \in L^{\infty}(0,1)$ and $\mu \in M_{B}^{+}(0,1)$ such that:

$$
\left\{\begin{array}{l}
\underline{v} \leq u \leq \bar{v} \quad \text { in } \quad(0,1) \\
-u^{\prime \prime} \leq \mu \quad \text { in } \quad \mathcal{D}^{\prime}(0,1) \\
-\bar{v}^{\prime \prime} \geq \mu \quad \text { in } \quad \mathcal{D}^{\prime}(0,1)
\end{array}\right.
$$

Then $u \in W_{l o c}^{1, \infty}(0,1)$, and

$$
\left|u^{\prime}(t)\right| \leq \frac{1}{d(t ; a, b)}\left(c(a, b)+\|\underline{v}\|_{L^{\infty}}+\|\bar{v}\|_{L^{\infty}}+\|\mu\|_{M_{B}}\right)
$$

for all $0<a<b<1$. Where $d(t ; a, b)=\min (b-t, t-a)$ and $c(a, b)$ is a constant depending on $a$ and $b$.

Lemma (2.6), will provide $W_{l o c}^{1, \infty}(0,1)$ estimates for the approximate solution $u_{n}$. But this estimate don't allow us to pass to the limit in the nonlinear terms. We need the strong convergence of $u_{n}$ in $W_{l o c}^{1, \infty}(0,1)$. We obtain this result from the following Lemma.

Lemma 2.7. Let $\left(u_{n}\right)_{n} \subset W_{0}^{1, \infty}(0,1)$ such that,

$$
\begin{gathered}
u_{n} \rightarrow u \quad \text { strongly in } L^{\infty}(0,1) \\
\left\{\begin{array}{l}
\underline{w} \leq u \leq u_{n} \leq \bar{w} \\
-u^{\prime \prime} \leq \mu \quad \text { in } \mathcal{D}^{\prime}(0,1) \\
-\bar{w}^{\prime \prime} \geq \mu \text { in } \mathcal{D}^{\prime}(0,1)
\end{array}\right.
\end{gathered}
$$

Then $u_{n}^{\prime} \rightarrow u^{\prime}$ strongly in $L_{\text {loc }}^{\infty}(0,1)$

Proof of lemma (2.6). Let $0<a<b<1$ and let $\varphi$ the capacity potential of $[a, b]$. The function $\theta=\bar{v}-u$ satisfies

$$
\left\{\begin{array}{c}
-\theta^{\prime \prime} \geq 0 \text { in } D^{\prime}(0,1) \\
\theta \in W_{l o c}^{1, \infty}(0,1) \cap L^{\infty}(0,1)
\end{array}\right.
$$

We have

$$
\int_{a}^{b}-\theta^{\prime \prime}=\int_{a}^{b}-\theta^{\prime \prime} \varphi \leq \int_{0}^{1}-\theta^{\prime \prime} \varphi=\int_{a}^{b} \theta \varphi^{\prime \prime} \leq c(a, b)+\|\bar{v}\|_{\infty}+\|\underline{v}\|_{\infty}
$$


Then

$$
\int_{a}^{b}-u^{\prime \prime}=\int_{a}^{b}-\theta^{\prime \prime}+\mu \leq c(a, b)+\|\bar{v}\|_{\infty}+\|\underline{v}\|_{\infty}+\|\mu\|_{M_{B}(0,1)}
$$

Using a similar technique, we deduce that for $a<x<y<b$, we have

$$
u^{\prime}(x)-u^{\prime}(y) \leq c(a, b)+\|\bar{v}\|_{\infty}+\|\underline{v}\|_{\infty}+\|\mu\|_{M_{B}(0,1)}
$$

Integrate w.r.t. $y$ over $(x, b)$, to find:

$$
\begin{array}{ccc}
(b-x) u^{\prime}(x) & \leq & (b-x)\left(c(a, b)+\|\bar{v}\|_{\infty}+\|\underline{v}\|_{\infty}+\|\mu\|_{M_{B}(0,1)}\right) \\
& \leq & c(a, b)+\|\bar{v}\|_{\infty}+\|\underline{v}\|_{\infty}+\|\mu\|_{M_{B}(0,1)}
\end{array}
$$

Integrate w.r.t. $x$ over $(a, y)$, to obtain

$(2.22) u(y)-u(a) \leq(y-a)\left(c(a, b)+\|\bar{v}\|_{\infty}+\|\underline{v}\|_{\infty}+\|\mu\|_{M_{B}(0,1)}\right)+(y-a) u^{\prime}(y)$.

Then we deduce the following uniform local estimate

$(2.23) \forall x \in[a, b], \quad\left\|u^{\prime}(x)\right\| \leq \frac{1}{d(x ; a, b)}\left(c(a, b)+\|\bar{v}\|_{\infty}+\|\underline{v}\|_{\infty}+\|\mu\|_{M_{B}(0,1)}\right)$

where $d(x ; a, b)=\min (x-a, b-x)$.

Proof of lemma (2.7). By lemma (2.6), we have $u \in W_{l o c}^{1, \infty}(0,1)$ and

$$
\forall x \in[a, b], \quad\left\|u^{\prime}(x)\right\| \leq c(a, b)+\|\bar{v}\|_{\infty}+\|\underline{v}\|_{\infty}+\|\mu\|_{M_{B}(0,1)} .
$$

We then consider the function $\theta_{n}=\bar{w}-u_{n}$ satisfies the equation

$$
\left\{\begin{array}{c}
-\theta_{n}^{\prime \prime} \geq 0 \text { in } \mathcal{D}^{\prime}(a, b) \\
0 \leq \theta_{n} \leq \bar{w}-u \in[0,1]
\end{array}\right.
$$

Let $\varphi$ the capacity potential of $[a, b]$, then we have:

$$
\begin{gathered}
\int_{a}^{b}\left|-\theta_{n}^{\prime \prime}\right|=\int_{a}^{b}-\theta_{n}^{\prime \prime}=\int_{a}^{b} \theta^{\prime \prime} \varphi \leq \int_{0}^{1}-\theta^{\prime \prime} \varphi \leq \int_{0}^{1}-\theta_{n} \varphi^{\prime \prime} \leq c(a, b) \\
\theta_{n}=\bar{w}-u_{n} \text { converge to } \bar{w}-u \text { in } L^{\infty}(0,1)
\end{gathered}
$$

and applying Ascoli's theorem, the lemma follows.

Proof of the theorem (2.3). First we prove

$$
\underline{w} \leq u_{n+1} \text { for all } n \geq 0
$$

Thanks to (2.11) and the definition of $\underline{w}$, we obtain

$$
-\left(u_{n+1}-\underline{w}\right)^{\prime \prime}+G\left(u_{n+1}^{\prime}\right)-G\left(\underline{w}^{\prime}\right) \geq 0 \text { in } \mathcal{D}^{\prime}(0,1)
$$

using (2.2) we then have

$$
\left\{\begin{array}{l}
-\left(u_{n+1}-\underline{w}\right)^{\prime \prime}+a_{n}\left(u_{n+1}-\underline{w}\right)^{\prime} \geq 0 \quad \text { in } \quad \mathcal{D}^{\prime}(0,1) \\
u_{n+1}-\underline{w} \in W_{0}^{1,1}(0,1) \\
a_{n}\left(u_{n+1}^{\prime}-w^{\prime}\right) \in L^{1}(0,1)
\end{array}\right.
$$

where $a_{n} \in \partial G\left(., u_{n+1}^{\prime}\right) \in L^{1}(0,1)$. Now we can apply lemma (2.5), therefore $\underline{w} \leq u_{n+1}$ in $[0,1]$ which proves $(2.28)$. 
Let us now prove by induction that

$$
u_{n+1} \leq u_{n} \leq \bar{w} \text { in }[0,1] \text { for all } n \geq 0
$$

For $n=0$, using (2.7), (2.11) we get

$$
\left\{\begin{array}{c}
\bar{w}-u_{1} \in W_{l o c}^{1,1}(0,1) \cap C_{0}[0,1] \\
-\left(\bar{w}-u_{1}\right) \geq 0 \text { in } \mathcal{D}^{\prime}(0,1)
\end{array}\right.
$$

Applying lemma (2.5) we have $\bar{w}-u_{1} \geq 0$. Let us assume $u_{n} \leq u_{n-1} \leq \bar{w}$, then from (2.11) and the monotonicity of $F$ in $r$, we have

$$
\left\{\begin{array}{l}
u_{n}-u_{n+1} \in W_{0}^{1,1}(0,1) ;\left(\bar{w}-u_{n}\right) \in W_{l o c}^{1,1}(0,1) \cap C_{0}[0,1] \\
-\left(u_{n}-u_{n+1}\right)^{\prime \prime}+G\left(u_{n+1}^{\prime}\right)-G\left(u_{n}^{\prime}\right) \geq 0 \text { in } \mathcal{D}^{\prime}(0,1) \\
-\left(\bar{w}-u_{n}\right)^{\prime \prime}-G\left(u_{n}^{\prime}\right) \geq 0 \quad \text { in } \mathcal{D}^{\prime}(0,1)
\end{array}\right.
$$

using now (2.3), (2.5) then we have from (2.33)

$$
\begin{gathered}
\left\{\begin{array}{l}
u_{n}-u_{n+1} \in W_{0}^{1,1}(0,1) \\
-\left(u_{n}-u_{n+1}\right)^{\prime \prime}+a_{n}\left(u_{n+1}-u_{n}\right)^{\prime} \geq 0 \quad \text { in } \mathcal{D}^{\prime}(0,1) \\
a_{n} \in \partial G\left(t, u_{n+1}^{\prime}\right) \in L^{1}(0,1)
\end{array}\right. \\
\left\{\begin{array}{l}
\bar{w}-u_{n} \in W_{l o c}^{1,1}(0,1) \cap C_{0}[0,1] \\
-\left(\bar{w}-u_{n}\right)^{\prime \prime} \geq 0 \text { in } \mathcal{D}^{\prime}(0,1)
\end{array}\right.
\end{gathered}
$$

Applying lemma (2.5), we deduce $u_{n+1} \leq u_{n} \leq \bar{w}$ in $[0,1]$ which proves $(2.31)$ by induction.

Employing lemma (2.6), we conclude that $u_{n}$ is bounded in $W_{l o c}^{1, \infty}(0,1) \cap C_{0}[0,1]$ independently of $n$. Therefore, there exists a subsequence, still denoted by $\left(u_{n}\right)$ for simplicity, such that $u_{n}$ converges to $u$ strongly in $L^{\infty}(0,1)$ if $n \rightarrow \infty$. Also $u_{n+1}^{\prime}$ converges to $u^{\prime}$ strongly in $L_{l o c}^{1}(0,1)$ and a.e. in $(0,1)$. Then from lemma $(2.6)$ we conclude that $u_{n+1}^{\prime}$ converges to $u^{\prime}$ strongly in $L_{l o c}^{\infty}(0,1)$, and

(2.36) $\left\|u_{n}^{\prime}\right\|_{L^{\infty}(a, b)} \leq K(a, b)\left(c(a, b)+\|\bar{w}\|_{L^{\infty}(0,1)}+\|f\|_{M_{B}}+\|\underline{w}\|_{L^{\infty}(0,1)}\right)$

where $K(a, b)=1 / \eta$ and $0<\eta<a<\eta+b<1$.

Since $G(t,$.$) and F(t,$.$) are continuous with respect the two last arguments,$ we have for all $0<a<b<1$

$$
G\left(t, u_{n+1}^{\prime}\right), F\left(t, u_{n}\right) \rightarrow G\left(t, u^{\prime}\right), F(t, u) \text { a.e. } t \in(0,1) .
$$

On the other hand, for a.e $t \in(a, b)$

$$
\mid G\left(t, u_{n+1}^{\prime}(t)\left|\leq \max _{|r| \leq C^{\prime}(a, b)}\right| G(t, r) \mid=\theta(t)\right.
$$

and

$$
\left|F\left(t, u_{n}(t)\right)\right| \leq \max _{|s| \leq \max \left(\|\bar{w}\|_{L^{\infty}(0,1)},\|\underline{w}\|_{L^{\infty}(0,1)}\right)}|F(t, s)|=\widehat{\theta}(t)
$$

and $\theta, \widehat{\theta} \in L_{l o c}^{1}(0,1)$ from (2.4). Using Lebesgue's dominate convergence Theorem (see [6]), we also have;

(2.40) $G\left(t, u_{n+1}^{\prime}\right), F\left(t, u_{n}\right) \rightarrow G\left(t, u^{\prime}\right), F(t, u)$ in $L^{1}(a, b)$ respectively 
Now, we can pass to the limit in $(2.11)$, and if $\varphi \in \mathcal{D}(0,1)$ with support of $\varphi \subset[a, b]$ then

$$
\begin{aligned}
0 & =\lim _{n \rightarrow \infty}\left\langle-u_{n+1}^{\prime \prime}+G\left(u_{n+1}^{\prime}\right)-F\left(u_{n}\right), \varphi\right\rangle \\
& =\left\langle-u^{\prime \prime}+G\left(u^{\prime}\right)-F(u), \varphi\right\rangle
\end{aligned}
$$

where $\langle.,$.$\rangle denotes the duality pairing between \mathcal{D}^{\prime}(0,1)$ and $\mathcal{D}(0,1)$. This completes the proof.

\section{Numerical method}

\subsection{Introduction}

In this section we present the numerical method to solve the equation (1.1). Formally the iterative method construct a sequence of numerical solutions of (2.11) in $H_{0}^{1}(0,1)$ with a first guess which is a super-solution of $(1.1)$, in our case a solution of the problem (2.7).

Then the algorithm can be formulated in the following way:

1) Find $\bar{w} \in H_{0}^{1}(0,1)$ such that:

$$
-\bar{w}^{\prime \prime}(t) \geq F(t, \bar{w})+f \text { in }(0,1)
$$

2) Given $u_{0}=\bar{w}$ we compute a sequence, $\left\{u_{n}\right\}_{n}$, solution in $H_{0}^{1}(0,1)$ of the non linear equation:

$$
-u_{n+1}^{\prime \prime}(t)+G_{n+1}\left(t, u_{n+1}^{\prime}\right)=F\left(t, u_{n}\right)+f \text { in }(0,1)
$$

Both problems (3.1) and (3.2) are nonlinear, and if (3.1) have a solution, in theorem 2.3 we prove that (3.2) have also a solution. Let us start by considering the numerical resolution of problem (3.1).

\subsection{Numerical resolution of equation (3.1)}

To solve the nonlinear equation (3.1), which presents some interesting difficulties, we consider the Newton method. We construct a sequence $\bar{w}^{k}$ such that $\left\{\bar{w}^{k}\right\}_{k}$ is a solution of a linear problem and $\bar{w}^{k}$ converges to $\bar{w}$.

Let $\bar{w}^{0}=0$, we define $\bar{w}^{k+1}=\bar{w}^{k}+\delta$ where $\delta$ is the solution of the following linear problem:

$$
\left\{\begin{array}{l}
-\delta^{\prime \prime}(t)-\frac{\partial F\left(t, \bar{w}^{k}\right)}{\partial r} \delta(t)=\left(\bar{w}^{k}\right)^{\prime \prime}(t)+F\left(t, \bar{w}^{k}\right)+f \quad \text { in } \quad(0,1) \\
\delta(0)=\delta(1)=0
\end{array}\right.
$$

Then at each iteration we have to solve the linear problem (3.3). To this aim we considered a weak formulation of the problem, finite element method and domain decomposition. 
3.3. Numerical resolution of problem (3.3) by domain decomposition.

To simplify the text we reformulate (3.3) in the following way: find $v \in H_{0}^{1}(a, b)$ such that:

$$
\left\{\begin{array}{c}
-v(t)^{\prime \prime}+c(t) v(t)=h(t) \text { in }(a, b) \\
v(a)=v(b)=0
\end{array}\right.
$$

where $h \in M_{B}(a, b)$, the set of finite measure in $(a, b)$, and $c(t) \in L^{2}(a, b)$, without any restriction in it sign. We assume $c_{\infty}=\|c\|_{L^{\infty}(a, b)}$ bounded.

In the previous section, Lemma 1, said that the problem (3.4) have a solution in a domain $(a, b)$ small enough.

If $V=H_{0}^{1}(a, b)$ then the weak formulation (3.4) reads:

$$
\text { find } v \in V: a(v, w)=(h, w) \forall w \in V
$$

where:

$$
\begin{aligned}
(v, w) & =\int_{a}^{b} u v d x \\
a(v, w) & =\left(v^{\prime}, w^{\prime}\right)+(c(t) v, w)
\end{aligned}
$$

Thanks to the Poincaré inequality we have:

$$
\left(v^{\prime}, w^{\prime}\right)=\left\|w^{\prime}\right\|_{L^{2}(a, b)}^{2} \geq \frac{c_{o}}{|b-a|}\|w\|_{L^{2}(a, b)}^{2}=\frac{c_{o}}{|b-a|}(v, w)
$$

and in the case of the bilinear form $a(w, v)$ we obtain:

$$
a(w, w)=\left(w^{\prime}, w^{\prime}\right)+(c w, w) \geq\left(\frac{c_{o}}{|b-a|}-c_{\infty}\right)(w, w)
$$

Then the bilinear form $a(w, v)$ should be coercive if $|b-a|<\frac{c_{0}}{c_{\infty}}$.

This remark are of great interest, because they can be exploited to obtain a numerical solution of (3.4) using a domain decomposition technique. In other words, this means that the domain partition should be determined by the behavior of $\left\|\frac{\partial F\left(\bar{w}^{k}\right)}{\partial r}\right\|_{\infty}$.

The aim of this section is to introduce the Schwarz overlapping domain decomposition method [15] applied to problem (3.4).

First we decompose $(a, b)$ in a set of $m$ overlapping sub-domains $\left(a_{i}, b_{i}\right)$ such that $(a, b)=\cup_{i=1}^{m}\left(a_{i}, b_{i}\right)$ and $\left(a_{i}, b_{i}\right) \cap\left(a_{i+1}, b_{i+1}\right) \neq \emptyset$ and satisfies :

$$
a_{i+1}<b_{i} \text { and }\left|b_{i}-a_{i}\right|<\min \left(\frac{c_{0}}{c_{\infty}}, \frac{\pi}{2 \sqrt{c_{\infty}}}\right)
$$

Then, if $v^{0}$ is an initialization function defined in $(a, b)$ and vanishing in $a$ and $b$ we define for $k \geq 0, m$ sequences $v_{i}^{k}, i=1, \ldots m$ solving the following problems:

$$
\left\{\begin{array}{c}
-\left(v_{1}^{k+1}\right)^{\prime \prime}(t)+c(t) v_{1}^{k+1}(t)=h \text { in }\left(0, b_{1}\right) \\
v_{1}^{k+1}(a)=0 ; v_{1}^{k+1}\left(b_{1}\right)=v_{2}^{k}\left(b_{1}\right)
\end{array}\right.
$$


for $i=2, \ldots m-1$

$$
\left\{\begin{array}{l}
-\left(v_{i}^{k+1}\right)^{\prime \prime}(t)+c(t) v_{i}^{k+1}(t)=h \text { in }\left(a_{i}, b_{i}\right) \\
v_{i}^{k+1}\left(a_{i}\right)=v_{i-1}^{k+1}\left(a_{i}\right) ; v_{i}^{k+1}\left(b_{i}\right)=v_{i+1}^{k}\left(b_{i}\right)
\end{array}\right.
$$

and

$$
\left\{\begin{array}{c}
-\left(v_{m}^{k+1}\right)^{\prime \prime}(t)+c(t) v_{m}^{k+1}(t)=h \text { in }\left(a_{m}, 1\right) \\
v_{m}^{k+1}\left(a_{m}\right)=v_{n-1}^{k+1}\left(a_{m}\right) ; v_{m}^{k+1}(b)=0
\end{array}\right.
$$

The variational formulation of the overlapping Schwarz method for the problem (3.3) can be stated as follows, set $V=H_{0}^{1}(a, b), V_{i}^{0}=H_{0}^{1}\left(a_{i}, b_{i}\right), i=1, \ldots m$ and

$$
a_{i}(v, w)=\int_{a_{i}}^{b_{i}} v^{\prime}(t) w^{\prime}(t)+c(t) v(t) w(t) d t
$$

Given $v^{0} \in V$, solve for each $k \geq 0$ :

$$
\begin{array}{r}
\eta_{1}^{k} \in V_{1}^{0}: a_{1}\left(\eta_{1}^{k}, w_{1}\right)=\left(f, w_{1}\right)-a_{1}\left(v^{k}, w_{1}\right) ; \forall w_{1} \in V_{1}^{0} \\
v^{k+\frac{1}{2}}=v^{k}+\tilde{\eta}_{1}^{k}
\end{array}
$$

for $i=2, \ldots m-1$

$$
\begin{array}{r}
\eta_{i}^{k} \in V_{i}^{0}: a_{i}\left(\eta_{i}^{k}, w_{i}\right)=\left(f, w_{i}\right)-a_{i}\left(v^{k}, w_{i}\right) ; \forall w_{i} \in V_{i}^{0} \\
v^{k+\frac{1}{2}}=v^{k}+\tilde{\eta}_{i}^{k} \\
\eta_{m}^{k} \in V_{m}^{0}: a_{i}\left(\eta_{m}^{k}, w_{m}\right)=\left(f, w_{m}\right)-a_{i}\left(v^{k}, w_{m}\right) ; \forall w_{m} \in V_{m}^{0} \\
v^{k+1}=v^{k}+\tilde{\eta}_{m}^{k}
\end{array}
$$

where $\tilde{\eta}_{i}^{k}$ denotes the extension of $\eta_{i}^{k}$ by 0 in $(a, b) \backslash\left(a_{i}, b_{i}\right)$.

\subsection{Numerical algorithm}

This subsection summarize the algorithm introduced in the previous subsection (3.1), (3.2) et (3.3).

1) First step: given $\bar{w}^{0}=0$, iteratively for $k=1$ until convergence we compute $\bar{w}^{k+1}=\bar{w}^{k}+\delta$ where at each iteration $\delta$ is the solution of the linear problem:

$$
\left\{\begin{array}{l}
-\delta^{\prime \prime}(t)-\frac{\partial F\left(t, \bar{w}^{k}\right)}{\partial r} \delta(t)=\left(\bar{w}^{k}\right)^{\prime \prime}(t)+F\left(t, \bar{w}^{k}\right)+f \quad \text { in } \quad(0,1) \\
\delta(0)=\delta(1)=0
\end{array}\right.
$$

At the end of the iterative process we obtain a discrete approximation of the supersolution $\bar{w}$. To solve at each iteration the linear problem (3.21) we consider the domain decomposition method introduced in the previous subsection. This step of the algorithm may be formulated as follows: 
a) We compute $c_{\infty}=\left\|\frac{\partial F\left(\bar{w}^{k}\right)}{\partial r}\right\|_{\infty}$.

Determine the $(0,1)$ overlapping sub-domains $\left(a_{i}, b_{i}\right)$ such that :

$(0,1)=\cup_{i=1}^{m}\left(a_{i}, b_{i}\right),\left(a_{i}, b_{i}\right) \cap\left(a_{i+1}, b_{i+1}\right) \neq \emptyset$ and satisfies :

$$
a_{i+1}<b_{i} \text { and }\left|b_{i}-a_{i}\right|<\min \left(\frac{c_{0}}{c_{\infty}}, \frac{\pi}{2 \sqrt{c_{\infty}}}\right)
$$

We denote $m$ the number of sub-domains $\left(a_{i}, b_{i}\right)$. In practice, the overlap used is never more than a fixed percentage, 10 to 20 percent of the width of the sub-domains.

b) Iteratively :

for $l=1, \ldots$ convergence

for $i=1, . ., m$

We solve the following sub-domains problems:

$$
\left\{\begin{array}{l}
-\left(\delta_{i}^{l}\right)^{\prime \prime}(t)-\frac{\partial F\left(t, \bar{w}^{k}\right)}{\partial r} \delta_{i}^{l}(t)=\left(\bar{w}^{k}\right)^{\prime \prime}(t)+F\left(t, \bar{w}^{k}\right)+f \quad \text { in } \quad\left(a_{i}, b_{i}\right) \\
\delta_{i}^{l}\left(a_{i}\right)=\delta_{i-1}^{l}\left(a_{i}\right), \delta_{i}^{l}\left(b_{i}\right)=\delta_{i+1}^{l-1}\left(b_{i}\right) \\
\text { where } \delta_{1}^{l}(0)=0, \text { and } \delta_{m}^{l}(1)=0
\end{array}\right.
$$

At each sub-domain $\left(a_{i}, b_{i}\right)$ we consider a finite element approximation method with $N_{i}$ elements. At the termination of the computation $\delta_{i}^{l}$ contains the approximate discrete solution for $\left(a_{i}, b_{i}\right) ; i=1, \ldots, m$. For the overlap regions $\left(a_{i}, b_{i}\right) \cap\left(a_{i+1}, b_{i+1}\right)$ we consider a average of the two solutions, since the two solutions, $\delta_{i}^{l}, \delta_{i}^{l+1}$, will both converge to the same values as the mesh is refined.

To determine when the Schwarz overlapping domain decomposition method has converged we require that the approximate solutions in the sub-domains or on the artificial boundaries change by less than a given tolerance from the previous iterations.

2) At this step for $u_{0}=\bar{w}$, iteratively for $n=1$, until convergence we solve the following non-linear problem obtained using the Yosida approximation of $G$ :

$$
\left\{\begin{aligned}
-u_{n}^{\prime \prime}(t)+G_{n}\left(t, u_{n}^{\prime}\right) & =F\left(t, u_{n-1}\right)+f \text { in }(0,1) \\
u_{n}(0) & =u_{n}(1)=0
\end{aligned}\right.
$$

At each n-step the problem (3.24) is solved using a Newton method.

The algorithm may be formulated in the following way:

$$
\begin{aligned}
& u_{0}=\bar{w} \\
& \text { for } n=1, \ldots \ldots \text { convergence } \\
& u_{n}^{0}=u_{n-1} \\
& \theta=0 \\
& \text { for } j=1, \ldots \text { convergence }
\end{aligned}
$$


$\theta$ is the solution of the linear problem

$$
\begin{gathered}
\left\{\begin{array}{ccc}
-\theta(t)^{\prime \prime}+\frac{\partial G_{n}\left(t, u_{n}^{j^{\prime}}\right)}{\partial r} \theta(t)^{\prime} & = & -G_{n}\left(t, u_{n}^{j^{\prime}}\right)+u_{n}^{j \prime \prime} \\
\theta(0)=\theta(1)=0 & + & F\left(t, u_{n-1}\right)+f
\end{array}\right. \\
u_{n}^{j+1}=u_{n}^{j}+\theta
\end{gathered}
$$

At termination of the j-loop $u_{n}=u_{n}^{j}$.

The discrete approximation of the solution of (1.1) is obtained at the termination of the n-loop.

\subsection{Convergence of the domain decomposition method}

To simplify, without lost of generality, we assume that we can consider a two domains decomposition $(a, b)=(a, \beta) \bigcup(\alpha, b)$ such that:

$$
\alpha<\beta \text { and }(\beta-a),(b-\alpha)<\min \left(\frac{c_{0}}{c_{\infty}}, \frac{\pi}{2 \sqrt{c_{\infty}}}\right)
$$

Then, if $v^{0}$ is an initialization function defined in $(a, b)$ and vanishing in $a$ and $b$ we define for $k \geq 0,2$ sequences $v_{i}^{k}, i=1,2$ solving the following problems:

$$
\left\{\begin{aligned}
-\left(v_{1}^{k+1}\right)^{\prime \prime}(t)+c(t) v_{1}^{k+1}(t) & =h \text { in }(a, \beta) \\
v_{1}^{k+1}(a)=0 ; v_{1}^{k+1}(\beta) & =v_{2}^{k}(\beta)
\end{aligned}\right.
$$

and

$$
\left\{\begin{array}{c}
-\left(v_{2}^{k+1}\right)^{\prime \prime}(t)+c(t) v_{2}^{k+1}(t)=h \text { in }(\alpha, b) \\
v_{2}^{k+1}(\alpha)=v_{1}^{k}(\alpha) ; v_{2}^{k+1}(b)=0
\end{array}\right.
$$

Now to prove the convergence of the Schwarz overlapping domain decomposition algorithm applied to problem (3.4) we consider two problems:

$$
\left\{\begin{array}{l}
-v_{1}(t)^{\prime \prime}+c(t) v_{1}(t)=h \in(a, \beta) \\
v_{1}(a)=0 ; v_{1}(\beta)=v_{2}(\beta)
\end{array}\right.
$$

and:

$$
\left\{\begin{array}{l}
-v_{2}(t)^{\prime \prime}+c(t) v_{2}(t)=h \text { in }(\alpha, 1) \\
v_{2}(\alpha)=v_{1}(\alpha), v_{2}(b)=0
\end{array}\right.
$$

Let $v$ be

$$
v=\left\{\begin{array}{l}
v_{1} \text { in }(a, \beta) \\
v_{2} \text { in }(\alpha, b)
\end{array} v_{1}=v_{2} \text { in }(\alpha, \beta)\right.
$$

With the restriction (3.26) we can suppose the existence of a solution of (3.29) in $C(a, \beta)$ and a solution of $(3.30)$ in $C(\alpha, b)$.

Theorem 3.1. Assume $a, b, \alpha$ and $\beta$ with the restriction (3.26). Then the sequence $v^{k}$ converges to $v$ in $C(a, \beta)$ and $C(\alpha, b)$. 
Proof:

Let $d^{k}=v_{1}^{k}-v$ in $(a, \beta)$ and $e^{k}=v_{2}^{k}-v$ in $(\alpha, b)$.

We prove the following inequality:

$$
\left\|d^{k+2}\right\|_{\infty} \leq \gamma\left\|d^{k}\right\|_{\infty} \text { and }\left\|e^{k+2}\right\|_{\infty} \leq \gamma\left\|e^{k}\right\|_{\infty}
$$

where $\gamma<1$.

The difference $d^{k}$ satisfies the following equation.

$$
\left\{\begin{array}{c}
-d^{k+1}(t)^{\prime \prime}+c(t) d^{k+1}(t)=0 \text { in }(a, \beta) \\
d^{k+1}(a)=0 \text { and } d^{k+1}(\beta)=v_{2}^{k}(\beta)-v(\beta)=e^{k}(\beta)
\end{array}\right.
$$

and $e^{k}$ satisfies a similar equation in $(\alpha, b)$ :

$$
\left\{\begin{array}{c}
-e^{k+1}(t)^{\prime \prime}+c(t) e^{k+1}(t)=0 \text { in }(a, \beta) \\
e^{k+1}(\alpha)=v_{1}^{k}(\alpha)-v(\alpha)=d^{k}(\alpha) \text { and } e^{k+1}(b)=0
\end{array}\right.
$$

If we consider the following equation:

$$
\left\{\begin{array}{l}
-\varphi(t)^{\prime \prime}-c_{\infty} \varphi(t)=0 \text { in }(a, \beta) \\
\varphi(a)=0 \text { and } \varphi(\beta)=\left|e^{k+1}(\beta)\right|
\end{array}\right.
$$

then $\varphi(t)=\left|e^{k+1}(\beta)\right| \frac{\sin \left(\sqrt{c_{\infty}}(t-a)\right)}{\sin \left(\sqrt{c_{\infty}}(\beta-a)\right)}$, this solution is unique and positive if $(\beta-a)<\frac{\pi}{2 \sqrt{c_{\infty}}}$. In that case $\|\varphi\|_{\infty}=\left|e^{k+1}(\beta)\right|$.

The difference $z=\varphi-d^{k+2}$ is the solution of:

$$
\left\{\begin{array}{c}
-z(t)^{\prime \prime}+c(t) z(t)=\left(c(t)+c_{\infty}\right) \varphi(t) \text { in }(a, \beta) \\
z(a)=0 \text { and } z(\beta)=\left|e^{k+1}(\beta)\right|-e^{k+1}(\beta)
\end{array}\right.
$$

Clearly $z \geq 0$ if $(\beta-a),(b-\alpha)<\min \left(\frac{c_{0}}{c_{\infty}}, \frac{\pi}{2 \sqrt{c_{\infty}}}\right)$. Then $d^{k+2} \leq \varphi \leq\left|e^{k+1}(\beta)\right|$.

If now $z=\varphi+d^{k+2}$ we have

$$
\left\{\begin{array}{c}
-z(t)^{\prime \prime}+c(t) z(t)=\left(c(t)+c_{\infty}\right) \varphi(t) \text { in }(a, \beta) \\
z(a)=0 \text { and } z(\beta)=\left|e^{k+1}(\beta)\right|+e^{k+1}(\beta)
\end{array}\right.
$$

Also $z \geq 0$ and $-\varphi \leq d^{k+2}(t), \forall t \in(a, \beta)$.

Then the inequality $\left\|d^{k+2}\right\|_{\infty} \leq\left|e^{k+1}(\beta)\right| \leq\left\|e^{k+1}\right\|_{\infty}$ holds.

To prove that $\left|e^{k+1}(\beta)\right| \leq \gamma\left\|\overline{d^{k}}\right\|_{\infty}$ with $\gamma<1$ we consider the equation:

$$
\left\{\begin{array}{c}
-\phi(t)^{\prime \prime}-c_{\infty} \phi(t)=0 \text { in }(\alpha, b) \\
\phi(\alpha)=\left|d^{k}(\alpha)\right| \text { and } \phi(b)=0
\end{array}\right.
$$

The solution of this equation is given by: $\phi(t)=\left|d^{k}(\alpha)\right| \frac{\sin \left(\sqrt{c_{\infty}}(b-t)\right)}{\sin \left(\sqrt{c_{\infty}}(b-\alpha)\right)}$. This solution is positive if $(b-\alpha)<\frac{\pi}{2 \sqrt{c_{\infty}}}$ and then $\phi(t) \geq\left|e^{k+1}(t)\right| \forall t \in(\alpha, b)$.

At this step we consider $z=\phi-e^{k+1}$. Then $z$ is the solution of:

$$
\left\{\begin{array}{c}
-z(t)^{\prime \prime}+c(t) z(t)=\left(c(t)+c_{\infty}\right) \phi(t) \text { in }(\alpha, b) \\
z(\alpha)=\left|d^{k}(\alpha)\right|-d^{k}(\alpha), \text { and } z(b)=0
\end{array}\right.
$$


Clearly $z \geq 0$ and then $\phi(t) \geq e^{k+1}(t)$ for all $t$ in $(\alpha, b)$.

If now we consider $z=\phi+e^{k+1}$ we have also $z \geq 0$ because $z(t)$ is the solution of the following equation:

$$
\left\{\begin{array}{c}
-z(t)^{\prime \prime}+c(t) z(t)=\left(c(t)+c_{\infty}\right) \phi(t) \text { in }(\alpha, b) \\
z(\alpha)=\left|d^{k}(\alpha)\right|+d^{k}(\alpha), \text { and } z(b)=0
\end{array}\right.
$$

Then $\left|e^{k+1}(t)\right| \leq \phi(t)$ in $(\alpha, b)$ and $\left|e^{k+1}(\beta)\right| \leq \phi(\beta) \leq \gamma\left|d^{k}(\alpha)\right|$ with $\gamma=\frac{\sin \left(\sqrt{c_{\infty}}(b-\beta)\right)}{\sin \left(\sqrt{c_{\infty}}(b-\alpha)\right.}$. The coefficient $\gamma$ is smaller than one only if $\alpha<\beta$.

In conclusion with the restriction (3.26) we have $\left\|d^{k+2}\right\|_{\infty}<\left\|d^{k}\right\|_{\infty}$.

Using the same technique we prove that $\left\|e^{k+2}\right\|_{\infty}<\left\|e^{k}\right\|_{\infty}$ if we have (3.26). First we prove that $\left\|e^{k+2}\right\|_{\infty} \leq\left|d^{k+1}(\alpha)\right|$. To this aim we consider the equation:

$$
\left\{\begin{array}{l}
-\lambda(t)^{\prime \prime}-c_{\infty} \lambda(t)=0 \text { in }(\alpha, b) \\
\lambda(\alpha)=\left|d^{k+1}(\alpha)\right| \text { and } \lambda(b)=0
\end{array}\right.
$$

The solution is given by $\lambda(t)=\left|d^{k+1}(\alpha)\right| \frac{\sin \left(\sqrt{c_{\infty}}(b-t)\right)}{\sin \left(\sqrt{c_{\infty}}(b-\alpha)\right)}$. This solution is positive if $b-\alpha<\min \left(\frac{c_{0}}{c_{\infty}}, \frac{\pi}{2 \sqrt{c_{\infty}}}\right)$.

If $z(t)=\lambda(t)+e^{k+2}(t)$ then $z(t)$ is the solution of the following equation:

$$
\left\{\begin{array}{c}
-z(t)^{\prime \prime}+c(t) z(t)=\left(c(t)+c_{\infty}\right) \lambda(t) \text { in }(\alpha, b) \\
z(\alpha)=\left|d^{k+1}(\alpha)\right|+d^{k+1}(\alpha) \text { and } z(b)=0
\end{array}\right.
$$

Clearly $z \geq 0$ if $(b-\alpha)<\min \left(\frac{c_{0}}{c_{\infty}}, \frac{\pi}{2 \sqrt{c_{\infty}}}\right)$.

If now $z(t)=\lambda(t)-e^{k+2}(t)$ then $z(t)$ is the solution of the following equation:

$$
\left\{\begin{array}{c}
-z(t)^{\prime \prime}+c(t) z(t)=\left(c(t)+c_{\infty}\right) \lambda(t) \text { in }(\alpha, b) \\
z(\alpha)=\left|d^{k+1}(\alpha)\right|-d^{k+1}(\alpha) \text { and } z(b)=0
\end{array}\right.
$$

then $z \geq 0$ if $(b-\alpha)<\min \left(\frac{c_{0}}{c_{\infty}}, \frac{\pi}{2 \sqrt{c_{\infty}}}\right)$.

It is an easy consequence that $\left|e^{k+2}(t)\right| \leq \lambda(t)$ and we conclude $\left\|e^{k+2}\right\|_{\infty} \leq$ $\left|d^{k+1}(\alpha)\right|$.

Now we prove that $\left|d^{k+1}(\alpha)\right| \leq \gamma\left|e^{k}(\beta)\right|$. To this aim we consider the following problem:

$$
\left\{\begin{array}{c}
-\eta(t)^{\prime \prime}-c_{\infty} \eta(t)=0 \text { in }(a, \beta) \\
\eta(a)=0 \text { and } \eta(\beta)=\left|e^{k}(\beta)\right|
\end{array}\right.
$$

The solution is given by $\eta(t)=\left|e^{k}(\beta)\right| \frac{\sin \left(\sqrt{c_{\infty}}(\beta-t)\right)}{\sin \left(\sqrt{c_{\infty}}(\beta-a)\right)}$. This solution is positive if $\beta-a<\min \left(\frac{c_{0}}{c_{\infty}}, \frac{\pi}{2 \sqrt{c_{\infty}}}\right)$. 
If $z(t)=\eta(t)+d^{k+1}(t)$ then it's the solution of the following equation:

$$
\left\{\begin{array}{c}
-z(t)^{\prime \prime}+c(t) z(t)=\left(c(t)+c_{\infty}\right) \eta(t) \text { in }(a, \beta) \\
z(a)=0 \text { and } z(\beta)=\left|e^{k}(\beta)\right|+e^{k}(\beta)
\end{array}\right.
$$

Then $z \geq 0$ if $(\beta-a)<\min \left(\frac{c_{0}}{c_{\infty}}, \frac{\pi}{2 \sqrt{c_{\infty}}}\right)$. In the same way if $z(t)=\eta(t)-d^{k+1}(t)$ it' the solution of:

$$
\left\{\begin{array}{c}
-z(t)^{\prime \prime}+c(t) z(t)=\left(c(t)+c_{\infty}\right) \eta(t) \text { in }(a, \beta) \\
z(a)=0 \text { and } z(\beta)=\left|e^{k}(\beta)\right|-e^{k}(\beta)
\end{array}\right.
$$

Then $z \geq 0$ if $(\beta-a)<\min \left(\frac{c_{0}}{c_{\infty}}, \frac{\pi}{2 \sqrt{c_{\infty}}}\right)$.

We obtain that $\left|d^{k+1}(t)\right| \leq \eta(t)$ for all $t \in(a, \beta)$ and then $\left|d^{k+1}(\beta)\right| \leq$ $\gamma\left|e^{k}(\beta)\right|$ with $\gamma=\frac{\sin \left(\sqrt{c_{\infty}}(\beta-\alpha)\right)}{\sin \left(\sqrt{c_{\infty}}(\beta-a)\right.}$. The coefficient $\gamma$ is smaller than one only if $\alpha<\beta$.

We conclude that the Schwarz overlapping domain decomposition method applied to the problem (3.4) converges.

\subsection{Numerical Results}

The algorithm introduced in the previous section has been implemented numerically for the model problem (1.3) with $p=q=3$ and $f=\delta_{\frac{1}{3}}, \delta_{x}$ denoting the Dirac measure giving unit mass to the point $x$.

$$
\left\{\begin{array}{l}
-u^{\prime \prime}(t)+\left|u^{\prime}(t)\right|^{q}=|u(t)|^{p}+\delta_{\frac{1}{3}} \text { in }(0,1) \\
u(0)=u(1)=0 \text { and } p=q=3
\end{array}\right.
$$

The number of sub-domains is not fixed, its changes at each iteration according to the criterion (3.26). In figure 1 it can be observed the shape of the super-solution and the solution when the algorithm converges with $m=10$ subdomains and $N_{i}=22$ finite element at each sub-domain.

To study the convergence history of the numerical simulation plotted in figure 1 we consider two steps. In the first step, where we compute a super-solution, we observe the evolution of the number of sub-domains: it goes from $m=2$ subdomains to $m=10$ sub-domains in five iterations according to criterion (3.26). Simulation stops after 17 iterations when the residual is of the order $10^{-11}$.

In the second step, starting with the super-solution computed in the previous step we perform nine iterations of the Yosida approximation described in section 2 and the simulation stops when the correction computed is in uniform norm of the order $10^{-11}$.

In figure 2 we consider the same example, but in that case we authorize a maximum of two sub-domains. Clearly, classical method fails to compute a solution.

In the next example we modified the function $G$ and $F$ in the following way:

$$
\left\{\begin{array}{l}
-u^{\prime \prime}(t)+\alpha(t)\left|u^{\prime}(t)\right|^{q}=\beta(t)|u(t)|^{p}+f \quad \text { in } \quad(0,1) \\
u(0)=u(1)=0
\end{array}\right.
$$




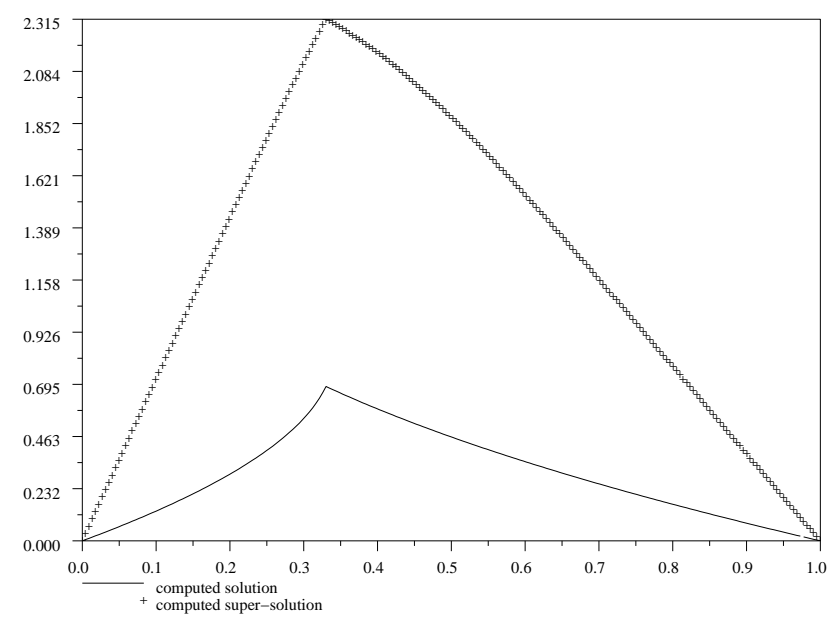

FIgURE 1. example $f=8 . \times \delta_{\frac{1}{3}}, \mathrm{~m}=10$

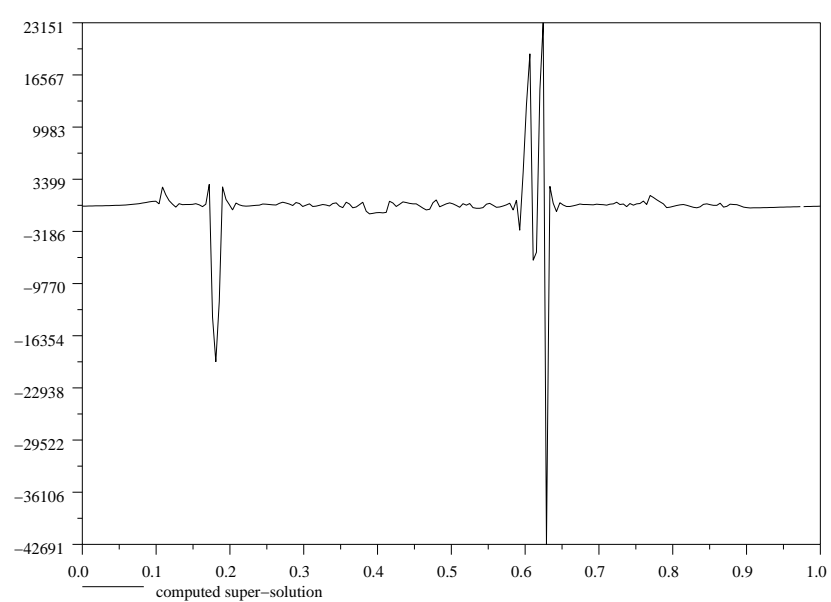

Figure 2. example $f=8 . \times \delta_{\frac{1}{3}}, \mathrm{~m}=2$ 


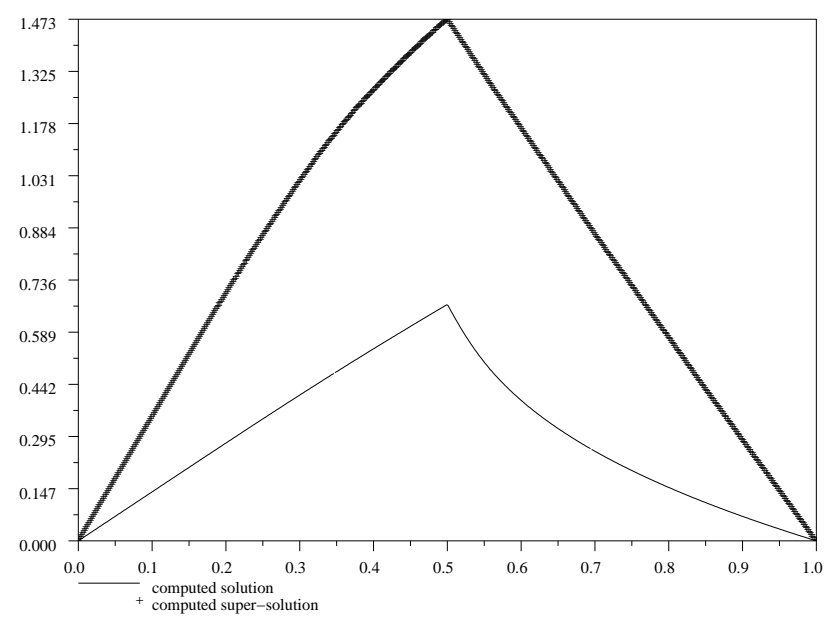

FiguRE 3. example $f=5 . \times \delta_{\frac{1}{2}}, \mathrm{~m}=7, \mathrm{p}=3, \mathrm{q}=4$

where $p=3, q=4$ and:

$$
\begin{aligned}
& \alpha(t)=\left\{\begin{array}{c}
0 \text { in }(0,0.5) \\
10 \times(t-0.5) \text { in }(0.5,1)
\end{array}\right. \\
& \beta(t)=\left\{\begin{array}{c}
36 \times(0.5-t) \text { in }(0,0.5) \\
0 \text { in }(0.5,1)
\end{array}\right.
\end{aligned}
$$

In figure 3 it can be observed the shape of the super-solution and the solution when the algorithm converges with $m=7$ sub-domains. In the first step, where we compute a super-solution, we can observe in figure 4 the evolution of the number of sub-domains required to satisfies criterion (3.26). At the first iteration the number of sub-domains are two, at the fifth iteration we have four sub-domains and after the eighteenth iteration we reach seven sub-domains, the algorithm converges at the twentieth iteration.

Starting with the super-solution computed in the first step we perform eleven iteration of the second step (2.11). For each iteration of the Yosida approximation (2.11) we perform about three step of the Newton method. The simulation stops when the correction computed is in uniform norm of order $10^{-11}$. 


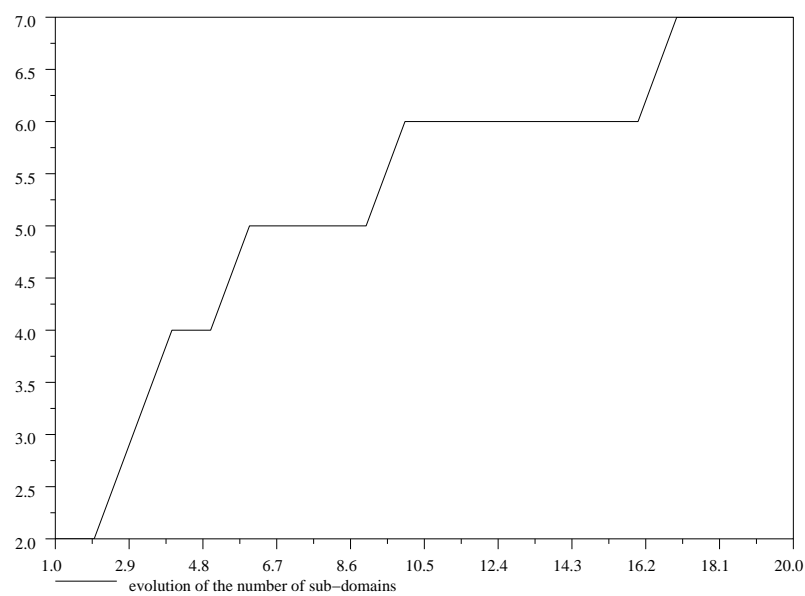

Figure 4. example $f=5 . \times \delta_{\frac{1}{2}}, \mathrm{~m}=7$

\section{References}

[1] N. Alaa, Etude d'équations elliptiques non-linéaires à dépendance convexe en le gradient et à données mesures, Thèse de Doctorat, Université de Nancy I, 1989.

[2] N. Alaa, M. Pierre, Weak solution of some quasilinear elliptic equations with data measures. SIAM J. Math. Anal. 24 (1993), 23-35.

[3] N. Alaa, M. Iguernane, Weak periodic solutions of some quasilinear parabolic equations with data measures. J. Inequal. Pure Appl. Math. 3 (2002).

[4] A. Bensoussan, L. Boccardo, F. Murat, On a nonlinear partial differential equation having natural growth terms and unbounded solution. Ann. Inst. Henri Poincaré $\mathbf{5}$ (1989), 347-364.

[5] L. Boccardo, F. Murat, J. P. Puel, Existence results for some quasilinear parabolic equations. Nonlinear Analysis Theory Method and Applications 13 (1989), 373-392.

[6] H. Brezis, Analyse fonctionnelle théorie et applications, Masson, 1983.

[7] P. Baras, M. Pierre, Critères d'existence de solutions positives pour des équations semi-linéaires. Annales Fourier Grenoble 24 (1984), 1985-2006.

[8] M. Dryja, B. F. Smith, O. B. Widlund,Schwarz analysis of iterative substructuring algorithms for elliptic problems in three dimensions. SIAM J. Numer. Anal. 31 (1994), 1662-1694.

[9] M. Dryja, O.B. Widlund, Domain decomposition algorithms with small overlap. SIAM J. Sci. Comput.,15 (1994), 604-620.

[10] M. Gadner, A waveform relaxation with overlapping splitting for reaction diffusion equations. Numerical Linear Algebra with Applications 6 Nr 2 (1993), 125-145. 
[11] S.A. Levin, Th.G. Hallam, L.J. Gross, Applied Mathematical Ecology. Biomathematics 18, Springer Verlag, 1989.

[12] P.L. Lions, Résolution de problèmes elliptiques quasilinéaires. Arch. Rational Mech. Anl. 74 (1980), 335-353.

[13] P.L. Lions, On the Schwarz alternating method I. In Roland Glowinski, Gene H. Golub, Gérard A. Meurant and J. Périaux editors, First International Symposium on Domain Decomposition Methods for Partial Differential Equations, SIAM Philadelphia (1988),1-42

[14] J.D. Murray, Mathematical Biology, Springer Verlag, 1993.

[15] A. Quarteroni, A. Valli, Domain decomposition Methods for Partial Differential Equations, Oxford Science Publications, 1999.

[16] B. Smith, P. Bjorstad, W. Gropp, Domain Decomposition, Parallel Multilevel Methods for Elliptic Partial Differential Equations, Cambridge University Press, 1996.

[17] P. Witomski, Sur la résolution numérique de quelques problèmes non-linéaires, Thèse d'Etat, Université Scientifique et Médicale de Grenoble, 1983.

\section{Acknowledgment}

This work was supported by the French Grant "Action Intégrée MA/02/33".

Département de Mathématiques et Informatique, Université des Sciences et Techniques Cadi Ayyad, B.P. 618, Guéliz,Marrakech,Maroc

E-mail address: alaa@fstg-marrakech.ac.ma

I.E.C.N., Université Henri Poincaré,, B.P. 239, 54506 Vandoeuvre lès Nancy, France

E-mail address: roche@iecn.u-nancy.fr 